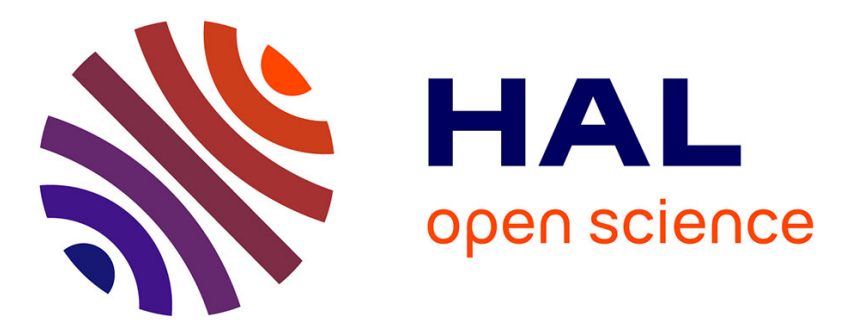

\title{
"Virtual" Signal Integrity Test on High-Speed Ethernet Cables in a Reverberation Chamber
}

\author{
Sahand Rasm, Guillaume Andrieu, Remi Tumayan, Alain Reineix
}

\section{To cite this version:}

Sahand Rasm, Guillaume Andrieu, Remi Tumayan, Alain Reineix. "Virtual" Signal Integrity Test on High-Speed Ethernet Cables in a Reverberation Chamber. 2020 International Symposium on Electromagnetic Compatibility - EMC EUROPE, Sep 2020, Rome (virtual), Italy. pp.1-4, 10.1109/EMCEUROPE48519.2020.9245721 . hal-03012444

\section{HAL Id: hal-03012444 \\ https://hal-unilim.archives-ouvertes.fr/hal-03012444}

Submitted on 19 Sep 2021

HAL is a multi-disciplinary open access archive for the deposit and dissemination of scientific research documents, whether they are published or not. The documents may come from teaching and research institutions in France or abroad, or from public or private research centers.
L'archive ouverte pluridisciplinaire HAL, est destinée au dépôt et à la diffusion de documents scientifiques de niveau recherche, publiés ou non, émanant des établissements d'enseignement et de recherche français ou étrangers, des laboratoires publics ou privés. 


\section{"Virtual" Signal Integrity Test on High-Speed Ethernet Cables in a Reverberation Chamber}

\author{
Sahand RASM \\ XLIM Laboratory, SRF axis, EMC team \\ Limoges, France \\ RENAULT, RF \& EMC department \\ Guyancourt, France \\ sahand.rasm@renault.com
}

\author{
Guillaume Andrieu \\ XLIM Laboratory, SRF axis, EMC team \\ Limoges, France \\ guillaume.andrieu@xlim.fr \\ Alain Reineix
XLIM Laboratory, SRF axis, EMC team
Limoges, France
alain.reineix@xlim.fr

\author{
Rémi Tumayan \\ RENAULT, RF \& EMC department \\ Guyancourt, France \\ remi.tumayan@renault.com
}

\begin{abstract}
In this paper, a "virtual" signal integrity method applied on twisted-wire pairs (shielded or not) is presented. The method is based on $S$-parameter measurements made in a reverberation chamber. From these balanced measurements, an impedance imbalance approximation is added in postprocessing through the introduction of a given common-mode rejection ratio. Then, the signal integrity can be tested for any level of the average electromagnetic field generated within the chamber. The method is illustrated in the simple case of a square useful signal in order to demonstrate the relevance of the method.
\end{abstract}

\section{INTRODUCTION}

In reason of the appearance of autonomous vehicles full of sensors, the volume of digital data to be transmitted within an automobile requires networks that support increasing data rates. It seems that twisted pairs of conductors inserted in Ethernet and LVDS cables will be the most commonly used cables for high-speed wired communications. Although highspeed networks solve the problem of data throughput, the study of their immunity is a new issue in the automotive industry. These types of studies are usually based on transfer impedance measurements at low frequencies [1]-[2] (when propagation effects along the cable are negligible) and shielding efficiency measurements at higher frequencies [3]. Immunity measurements on cables is generally made through the calculation of the induced signals (current and voltage) on the cable by an external disturbance [4] and do not involve checking the quality of the transmitted signal. To go further, a "virtual" method for verifying the signal integrity of highspeed networks is proposed in this paper. The method is based on the measurement of S-parameters in a mechanically stirred reverberation chamber (RC). It is shown that it is possible to predict the integrity of data transmission for a cable under any electromagnetic disturbance occurring at a given frequency.

\section{METHOD DESCRIPTION}

\section{A. Experimental setup}

The proposed method consists of $S$-parameters measurement between an antenna (port 1) and an under-test twisted pair (shielded or unshielded) Ethernet cable inserted in a reverberation chamber for a given number $N$ of positions of the rotating mode stirrer. The experimental setup is presented in Fig. 1. Ports 2 and 3 correspond to the termination (with respect to the ground) of each conductor of the twisted pair at one side of the cable, the conductors being connected to a $50 \Omega$ load on the other side of the cable.

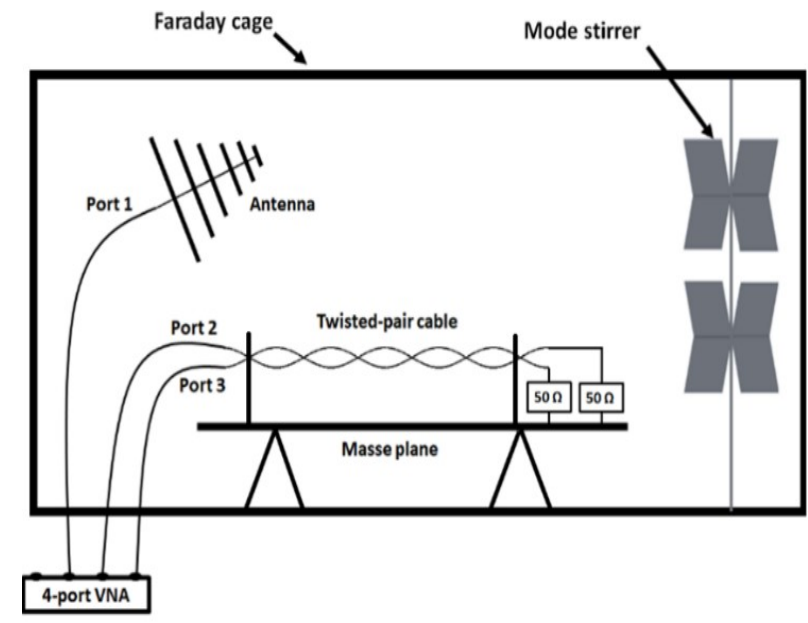

Fig. 1. Schematic description of the experimental setup.

\section{B. Principle of the method}

The different steps of the method are presented successively in this section.

\section{1) Calculation of $\left\langle\left|\mathrm{E}_{\mathrm{T}}\right|\right\rangle$}

The parameter $S_{11}$ is used to calculate the quality factor $Q$ of the RC [5]:

$$
Q=\left\langle\left|S_{11}-\left\langle S_{11}\right\rangle\right|^{2}\right\rangle \frac{Z_{0} \omega \varepsilon V}{\left(\frac{\lambda^{2}}{4 \pi}\right)\left(1-\left|\left\langle S_{11}\right\rangle\right|^{2}\right)^{2} \eta^{2}}
$$

where $Z_{0}$ is the wave impedance, $\omega$ the pulsation, $\lambda$ the wavelength, $\eta$ the antenna efficiency, $\varepsilon$ the dielectric permittivity of the propagation medium and $V$ the RC volume.

Knowing the RC quality factor $Q$, it is therefore possible to calculate the average total electric field strength $\left\langle\left|E_{T}\right|\right\rangle$ in the RC [6] for any power accepted by the antenna $P_{i n j}$.

$$
\left\langle\left|E_{T}\right|\right\rangle=\sqrt{\frac{Q P_{i n j}}{\omega \varepsilon V}}
$$




\section{2) Common mode induced voltage}

The parameters $S_{21}$ and $S_{31}$ measured allow the common mode voltages $V_{\mathrm{mc} 1} \& V_{\mathrm{mc} 2}$ generated on each of the 2 conductors of the twisted pair by the electromagnetic disturbance produced in the $\mathrm{RC}$ to be calculated. In the case of a twisted wire pair, the useful signal is generally transmitted in differential mode. The different ports of the measurement are illustrated in Figure 2 according to the S-parameter theory:

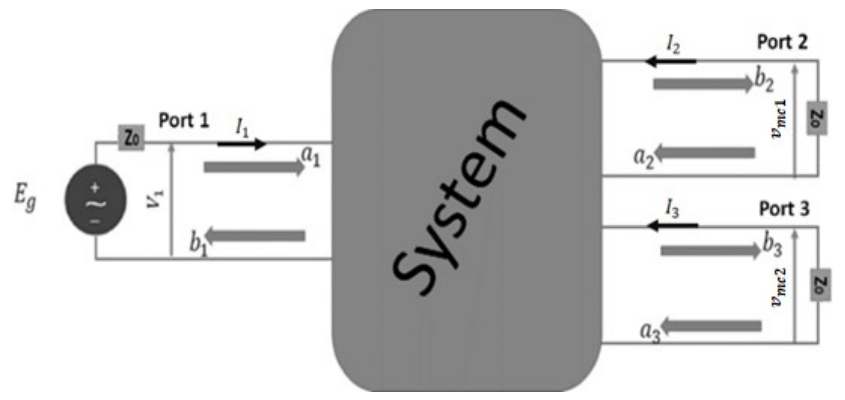

Fig. 2. Diagram of the measurement setup according to S-parameter theory

Common-mode $(\mathrm{CM})$ voltages $\mathrm{V}_{\mathrm{mc} 1} \& \mathrm{~V}_{\mathrm{mc} 2}$ can be written as follows [7]

$$
\left\{\begin{array}{l}
V_{m c 1}=\frac{S_{21} E_{g}}{2} \\
V_{m c 2}=\frac{S_{31} E_{g}}{2}
\end{array}\right.
$$

where $E_{g}$ is the internal voltage source of port 1 related to the power accepted by the antenna $P_{\text {inj }}$

$$
E_{g}=\sqrt{\frac{8 Z_{0} P_{i n j}}{1-\left|S_{11}\right|^{2}}}
$$

According to the measurement configuration shown in Fig.1, the impedances used at the end of each conductor of the twisted pair are equal. Due to this symmetry, the induced common mode voltages $\mathrm{V}_{\mathrm{mc} 1} \& \mathrm{~V}_{\mathrm{mc} 2}$ are similar and the differential signal related to the common mode disturbance $\mathrm{V}_{\mathrm{md}}=\mathrm{V}_{\mathrm{mc1}}-\mathrm{V}_{\mathrm{mc} 2}$ is almost equal to zero. In a real system, these impedances are often unbalanced which induces an important conversion of the parasitic common mode signal to the differential mode (DM). Of course, the amplitude of this $\mathrm{CM} / \mathrm{DM}$ conversion can have important effect on the possible perturbation of the useful signal due to the EM disturbance.

\section{3) Common mode rejection rate}

The CM/DM conversion at the input of the equipment connected at the end of the twisted pair under test can be represented by the common mode rejection ratio (CMRR) [8]. This parameter defines a relationship between the common mode and differential mode induced voltages and therefore quantifies the $\mathrm{CM} / \mathrm{DM}$ conversion as shown in Fig.3. The CMRR (in $\mathrm{dB}$ ) is defined as below

$$
C M R R=20 \log \left(\frac{V_{m c 1}+V_{m c 2}}{2 V_{m d}}\right)
$$

\section{4) Induced voltage in differential mode}

By applying a given CMRR, we can calculate the differential voltage $V_{\mathrm{md}}$ induced at the end of the under-test twisted wire pair (shielded or unshielded) by the EM disturbance produced in the $\mathrm{RC}$ for a given power accepted by the antenna

$$
V_{m d}=\frac{V_{m c 1}+V_{m c 2}}{2 \times 10\left(\frac{C M R R}{20}\right)}
$$

It is understood here that a high value of CMRR represents a good balance of the common mode impedances and therefore a low disturbance in the differential mode. The S-parameter theory makes it possible to calculate $\mathrm{V}_{\mathrm{md}}\left(f_{p}\right)$ obtained for each position of the mode stirrer and for any power accepted by the antenna or even according to equation (3) for any value of the average total electric field $\left\langle\left|E_{T}\right|\right\rangle$ in the RC. This reasoning is valid for any frequency $f_{p}$ for which the S-parameters have been previously measured.
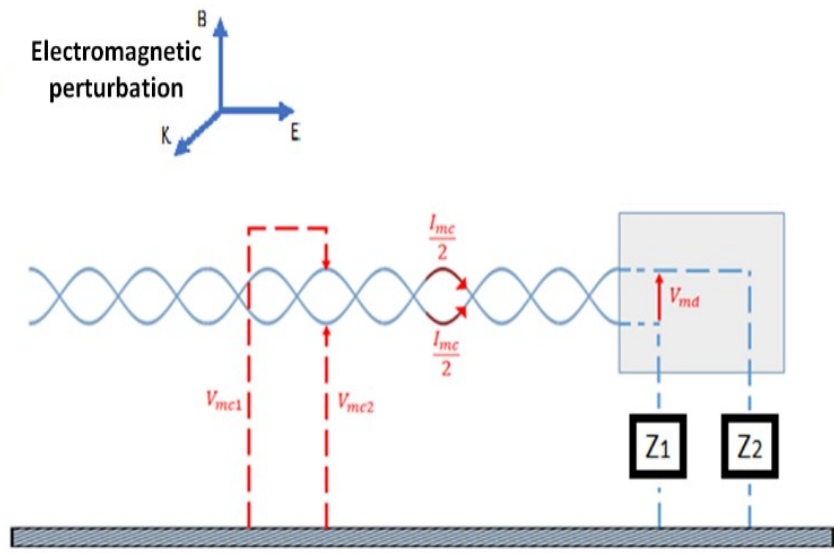

Fig. 3. Common mode rejection rate for a twisted pair

\section{5) Virtual test of signal integrity}

The last step of the method is the "virtual" verification of the quality of the transmitted signal in post-processing. To do this, the interfering sinusoidal signal at the frequency $\mathrm{V}_{\mathrm{md}}\left(f_{p}\right)$ is added to the "fictitious" useful signal in the time domain. The sinusoidal signal related to the perturbation is characterized by its peak amplitude $A_{p}$ and its frequency $f_{p}$. We see here a parallel with the traditional radiated immunity test where the electromagnetic disturbance is generally carried out in $\mathrm{CW}$ (constant wave) mode, i.e. for a single disturbance frequency.

This operation being performed in post-processing is therefore applicable to any type of time-domain useful signal. Once the transmitted signal (i.e. the sum of the useful and the parasitic signal) is calculated, it can be directly compared with the undisturbed useful signal. The result of this comparison makes it possible to study the integrity of the transmitted signal through the EM disturbance generated in the $\mathrm{RC}$, for each position of the mode stirrer and any considered frequency.

The analysis depends on the characteristics of the useful signal. It means that different metrics can be used to judge the quality of the transmitted signal. For instance, it can vary from a simple comparison between the amplitudes of the 
useful signal and the sinusoidal perturbation until a bit error rate study (at the price of course of a much more complex post-processing program).

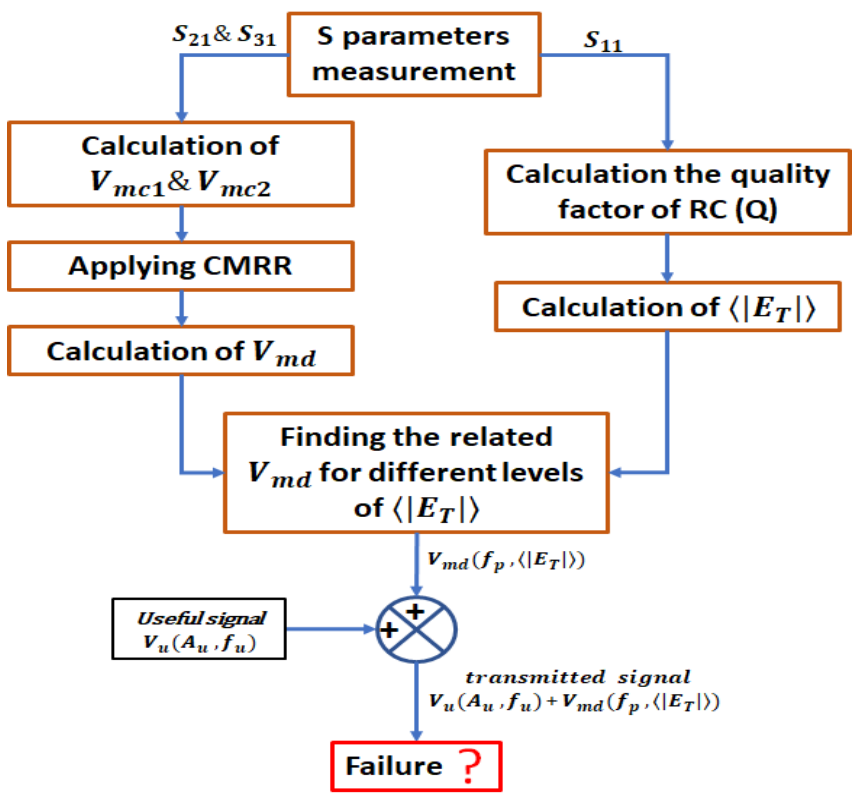

Fig. 4. Flowchart presenting the different steps of the method.

\section{RESULTS ON A SQUARE USEFUL SIGNAL}

\section{A. Calculation of $\left\langle\left|E_{T}\right|\right\rangle$}

To illustrate the method, a setup based on the experimental setup shown in Fig.1 has been installed in the $\mathrm{RC}$ of the XLIM laboratory having the following dimensions: $3.57 \mathrm{~m}$ long, $2.45 \mathrm{~m}$ wide and $2.46 \mathrm{~m}$ high corresponding to a volume of about $21.5 \mathrm{~m}^{3}$. The used antenna is a log-periodic one covering a frequency band from $200 \mathrm{MHz}$ to $2 \mathrm{GHz}$. In order to deal with many samples, the measurement was carried out for 360 positions of the mode stirrer (rotation of $1^{\circ}$ between two successive positions). The measurements have been performed for two types of Ethernet cables of $1 \mathrm{~m}$ length. The first is an unshielded twisted pair (UTP) while the second cable is a double shielded twisted pair (F/STP) covered by an aluminum foil and a braided shielding also made of aluminum. After the measurement of the $S$-parameters, the RC quality factor shown in Fig. 5 has been calculated using (1).

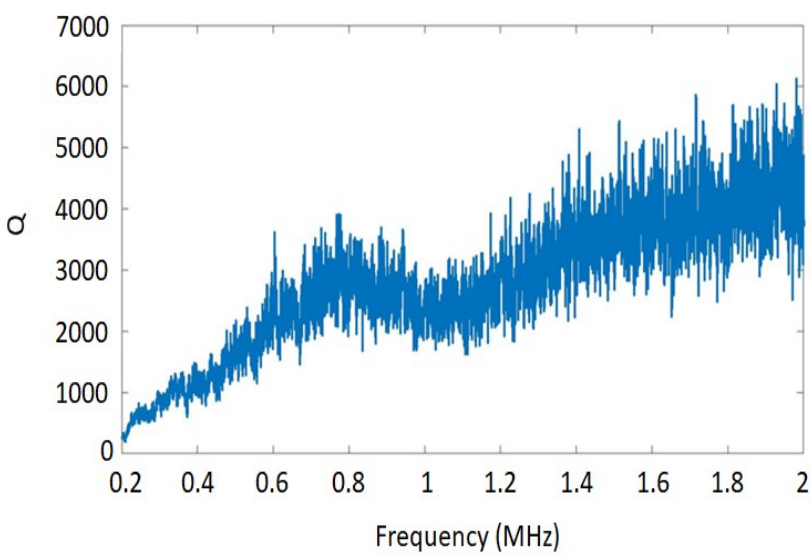

Fig. 5. Quality factor of the XLIM RC as a function of frequency
The RC XLIM is considered to be well stirred [2] for the frequencies above $400 \mathrm{MHz}$ when left empty (no absorber inserted inside). Using equation (3), the needed $\mathrm{P}_{\text {inj }}$ to obtain $\left\langle\left|E_{T}\right|\right\rangle=100 \mathrm{~V} / \mathrm{m}$ is calculated.

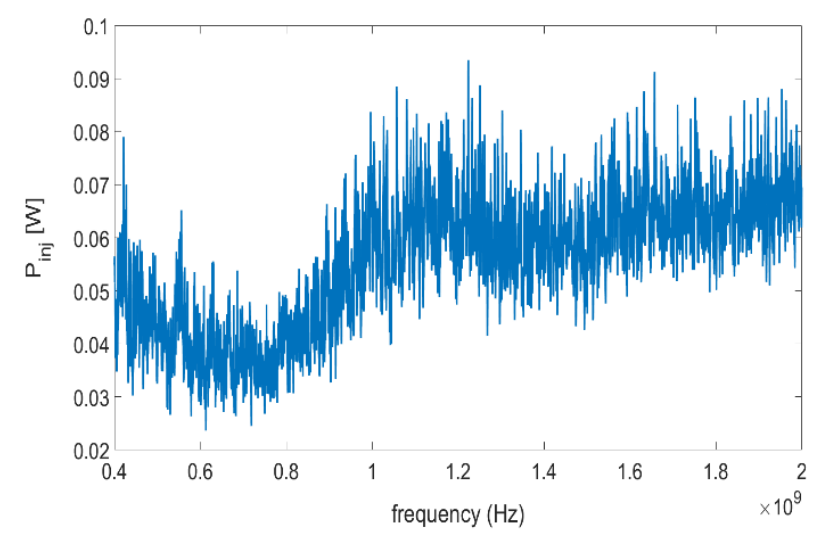

Fig. 6. Injected power $\mathrm{P}_{\text {inj }}(\mathrm{f})$ to get $\left\langle\left|E_{T}\right|\right\rangle=100 \mathrm{~V} / \mathrm{m}$ in the XLIM RC

\section{B. Calculation of $V_{m d}$}

Figure 7 shows the differential voltage obtained for the unshielded cable for different values of the CMRR for a $\left\langle\left|E_{T}\right|\right\rangle=100 \mathrm{~V} / \mathrm{m}$. As mentioned before, it is also important to note that $\mathrm{V}_{\mathrm{md}}(\mathrm{f})$ can be calculated for each position of the mode stirrer.

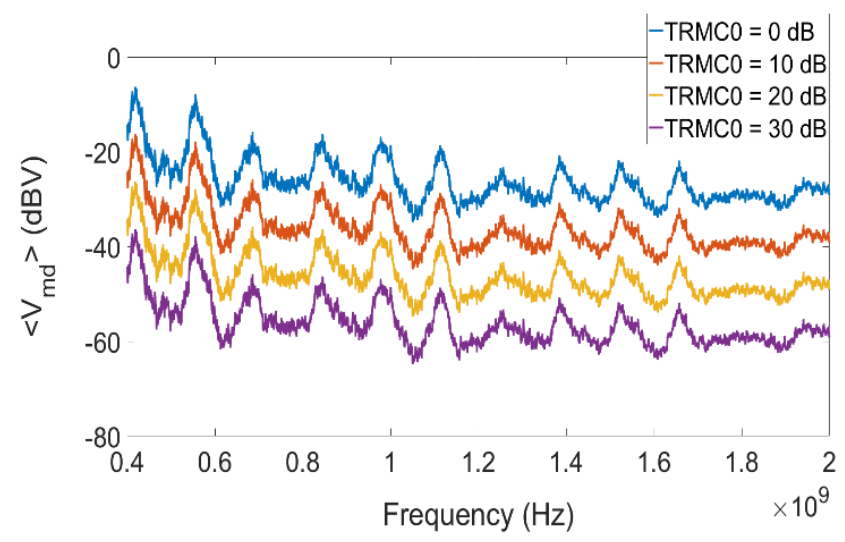

Fig. 7. Differential voltage induced on the unshielded cable for $\left\langle\left|E_{T}\right|\right\rangle=100 \mathrm{~V} / \mathrm{m} \mathrm{dBm}$ (for one mode stirrer position) and for different values of CMRR.

\section{Virtual signal integrity test}

The last step of the method is applied for the case of a square useful signal as shown in blue in Figure 8. This signal is defined in our work by a "high" state of value $A_{u}$ and a "low" state of value $-A_{u}$ and a repetition frequency of $f_{u}$. Figure 8 also represents the parasitic signal defined by its peak amplitude $A_{p}$ and its frequency $f_{p}$ (with $f_{p}>f_{u}$ in the figure) as well as the total amplitude of the so-called "disturbed" signal representing the sum of the two signals. The "failure" of the communication is then defined for each position of the mode stirrer and each value of considered $\left\langle\left|\mathrm{E}_{\mathrm{T}}\right|\right\rangle$ in the following way: if during a "high" state of the useful signal, the transmitted signal is less than 0 at a given moment (and inversely for a low level), a failure in the signal integrity is considered. The "failure rate" is defined, for a 
given $\left\langle\left|\mathrm{E}_{\mathrm{T}}\right|\right\rangle$, as the number of mode stirrer positions for which the failure occurs divided by the total number of considered mode stirrer positions.

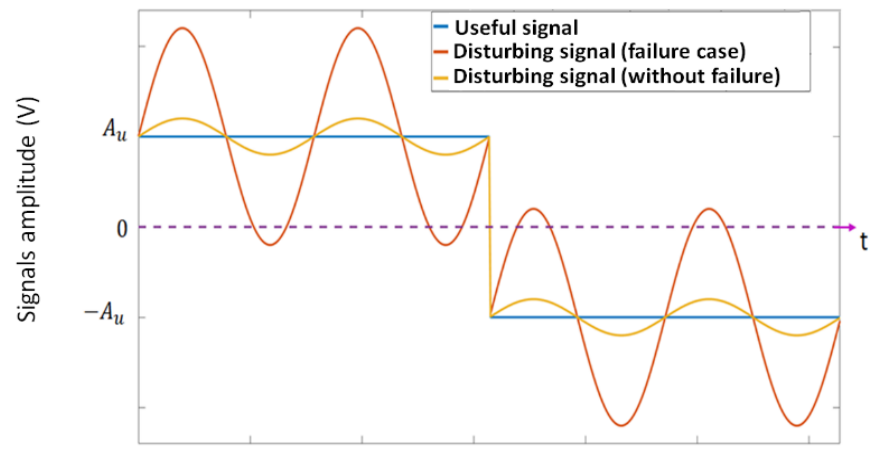

Fig. 8. Failure criterion in the case of a square useful signal.

Figure 9 shows the failure rate calculated for both cables as a function of the level of $\left\langle\left|E_{T}\right|\right\rangle$, fictitiously applied in the RC for a CMRR of $10 \mathrm{~dB}$. The amplitude $\mathrm{A}_{\mathrm{u}}$ of the considered useful signal is equal to $2 \mathrm{~V}$, its repetition frequency $f_{u}$ is $100 \mathrm{MHz}$ and the frequency of the disturbing signal $f_{p}$ is $1 \mathrm{GHz}$

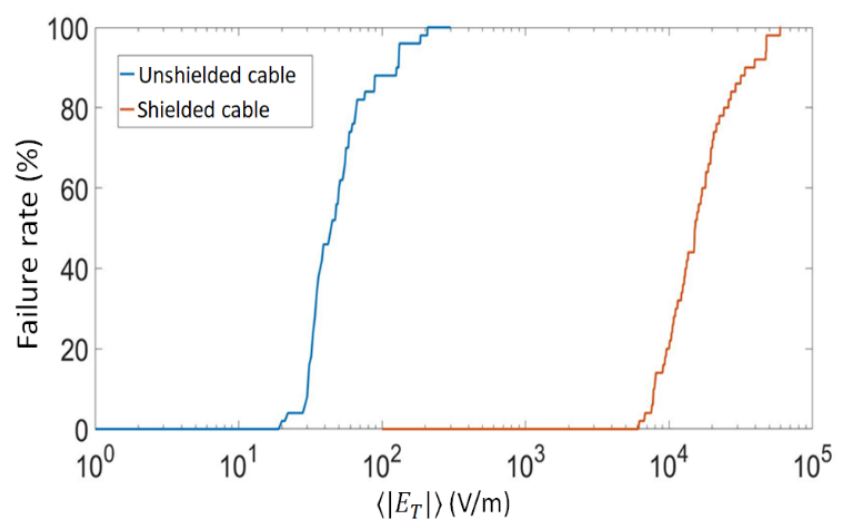

Fig. 9. Failure rate calculated for both cables $(f u=100 \mathrm{MHz}, f p=1 \mathrm{GHz}$, $\mathrm{TRMC}=10 \mathrm{~dB}, \mathrm{Au}=2 \mathrm{~V})$ as a function of $\left\langle\left|\mathrm{E}_{\mathrm{T}}\right|\right\rangle$ generated in the RC.

In figure 9, the effect of the shielding on the signal integrity of the useful signal is clearly evidenced. A perfect transmission of the useful signal (i.e. a failure rate of $0 \%$ ) is obtained until $\left\langle\left|\mathrm{E}_{\mathrm{T}}\right|\right\rangle\left\langle 20 \mathrm{~V} / \mathrm{m}\right.$ and $\left\langle\left|\mathrm{E}_{\mathrm{T}}\right|\right\rangle<5000 \mathrm{~V} / \mathrm{m}$ for the unshielded and the shielded cable respectively.

\section{CONCLUSION}

This paper presents an original method for virtually testing the integrity of information transmission on shielded and unshielded twisted pairs based on S-parameter measurements performed in an RC. Due to the S-parameter principle, the analysis performed in post-processing can be performed for any level of the mean total electric field present in the RC. Apart from the (rapid) S-parameter measurements, the rest of the method is carried out in post-processing, which makes it applicable for any type of digital communication.

\section{REFERENCES}

[1] Metallic Communication Cable TestMethods - Part 415: Electromagnetic Compatibility (EMC) - Test Method for Measuring Transfer Impedance and Screening Attenuation - or Coupling Attenuation With Triaxial Cell, IEC 62153-4-15:2015, Dec. 4, 2015.

[2] B. Demoulin and L. Kone, "Shielded cables transfer impedance measurement, "IEEE EMC Soc. Newslett., vol. 2010, no. 227 , pp. 38-45, 2010.

[3] Z. Chérif, G. Andrieu, G. Alberto, N. Ticaud, C. Jullien, J. Genoulaz, A. Dieudonné, "Transfer Impedance Measurement of Shielded Cables Through Localized Injection,” IEEE Trans. Electromagn. Compat., vol. 60, no. 4, pp. 1018-1021, Aug. 2018.

[4] F. Grassi, S. Pignari, "Current Injection in Twisted Wire Pairs With Not Perfectly Balanced Terminations," IEEE Trans. Electromagn. Compat., vol. 55, no. 6, pp. $1293-$ 1301, Dec. 2013.

[5] P. Besnier, C. Lemoine, J. Sol, "Various estimations of composite q-factor with antennas in a reverberation chamber," in Proc. 2015 IEEE Int. Symp. Electromagn. Compat., Aug. 2015, pp. 1223-1227.

[6] G. Andrieu, N. Ticaud, F. Lescoat, L. Trougnou, "Fast and Accurate Assessment of the "Well Stirred Condition" of a Reverberation Chamber from S11 Measurements," IEEE Trans. Electromagn. Compat., vol. 61, no. 4, pp. 974-982, Aug. 2019.

[7] Pozar, David M, Microwave engineering, Hoboken, NJ: Wiley, New York, 2012.

[8] H. W. Ott, Noise Reduction Techniques in Electronic Systems, 2nd ed. New York, NY, USA: Wiley, 1988. 